\title{
High-Resolution Incoherent Imaging in a Cs Corrected Electron Microscope: A New Tool for High -Resolution Electron Tomography in Life Science
}

\author{
Dirk Van Dyck ${ }^{1}$, Ivan Lobato Hoyos ${ }^{1}$, Uwe Lücken ${ }^{2}$, Holger Stark $^{3}$ \\ 1. University of Antwerp, EMAT, Department of Physics, Antwerp, Belgium. \\ 2. FEI Company, Eindhoven, Netherlands. \\ 3. MPI for biophysical Chemistry, Am Fassberg 11, 37077 Goettingen, Germany
}

In the world of single particle cryo-EM, biological objects are considered as phase objects that can only be studied using phase contrast microscopy. The usual way to get phase contrast in EM is by compensating the spherical aberration Cs with negative defocus (Scherzer focus) so as to create a passband of spatial frequencies for which the phase shift is close to $90^{\circ}$. However, in such a passband the phase transfer at the low spatial frequencies is very poor and is a problem for biological objects as information about their shape is in this range. A possible solution is to develop a phase plate that is able to shift the phase of the central beam over $90^{\circ}$ with respect to the diffracted beams but thus far, no workable electrostatic phase plate has been demonstrated. A more practical way to obtain phase contrast in the low spatial frequency range is by using a very large defocus and by compensating for the missing gaps in the transfer function by combining several defocus values. However, at large defocus, the spatial incoherence of the microscope effectively limits resolution to about $0.4 \mathrm{~nm}$. Therefore, it is generally believed that the use of a Cs corrector, in this specific case is of limited value for single-particle Cryo-EM ("Cs is our friend not our enemy"). However preliminary experiments of Cryo-EM with a Cs corrector seem to indicate that one can get better contrast with less defocus as shown in figure 1 for an HppS protein of $125 \mathrm{kDa}$ because its small size is difficult to image under the usual native cryo-EM conditions. Our recent work on incoherence in EM [1] suggested that this dark contrast could be due to thermal diffuse scattered (TDS) electrons scattering into the background. And, contrary to the common belief, the contribution of TDS electrons can be relatively large in soft matter because it is not only a function of the atomic number of the atoms but also of their mean square displacement and it can reach levels of the order of 10 to $40 \%$ of the total scattered intensity [1].

This had then inspired us to develop an alternative HREM imaging technique for biological objects that exploits the properties of TDS electrons. TDS scattering is incoherent and thus does not interfere with the central beam so that it generates amplitude contrast. It is maximal at a spatial frequency, which is inversely related to the mean square displacement of the atom, which in soft matter is about $1.0 \AA^{-1}$. At this spatial frequency, the phase transfer function can be made flat by combining a very low Cs with a small underfocus so as to get the highest resolution. Furthermore it is linear in the "mass-thickness" and easy to interpret and so that is it very suited for tomography. In this abstract we will outline the basic principles and support this by simulations. The details will be published in a forthcoming paper [3].

\section{High resolution incoherent imaging}

In a real object, the atoms vibrate around an average position. This is usually described by a Debye Waller factor, which reduces the scattering at large angles with only a minor influence on the image contrast. However as shown in [1] this is a misconception. Every electron sees a still atom with a sharply peaked electrostatic potential at the nucleus, which scatters at very large angles. Because of the phase oscillations of the transfer function at large spatial frequencies, this information is usually delocalized in the image - but using a low Cs and defocus this delocalization can be minimized by shifting the passband of the transfer function to the spatial frequency of the TDS signal. In addition, the averaging over the vibrating atom positions has to be done at the level of the detection of the image. If we assume that the atom motions are uncorrelated (Einstein model) the TDS contribution of each atom is incoherently (independently) added to the final image [3] which simplifies the 
interpretation of the high resolution images and it is very suitable for tomographic reconstruction algorithms. It is also possible to correct for slight differences in defocus of the atoms in the 3D object [3]. However the simulation of such imaging conditions requires the averaging of images calculated for different atom positions so that the computational requirements are comparable to the tedious frozen phonon calculations in HAADF STEM.

\section{Dark-field incoherent imaging}

It is possible to select only the TDS signal by using an annular dark field aperture [2], which matches the TDS contribution and by optimizing Cs and defocus so that the phase is approximately constant over this window. In a sense the method is then comparable to HAADF STEM but with all the advantages of HREM. Figure 2 shows the simulations of rotationally averaged power spectra of diffraction pattern and HREM image and figure 3 a simulation of the dark field images of the HbpS protein that proves that the incoherent imaging mode yields both high contrast and atomic resolution.

\section{References}

[1] D. Van Dyck, Ultramicroscopy 111 (2011), 894-900.

[2] S. Bals, R. Kilaas, C. Kisielowski, Ultramicroscopy 104 (2005), 281-289.

[3] Dirk Van Dyck, Ivan Lobato Hoyos, Uwe Lücken, Holger Stark ${ }^{2}$ to be published

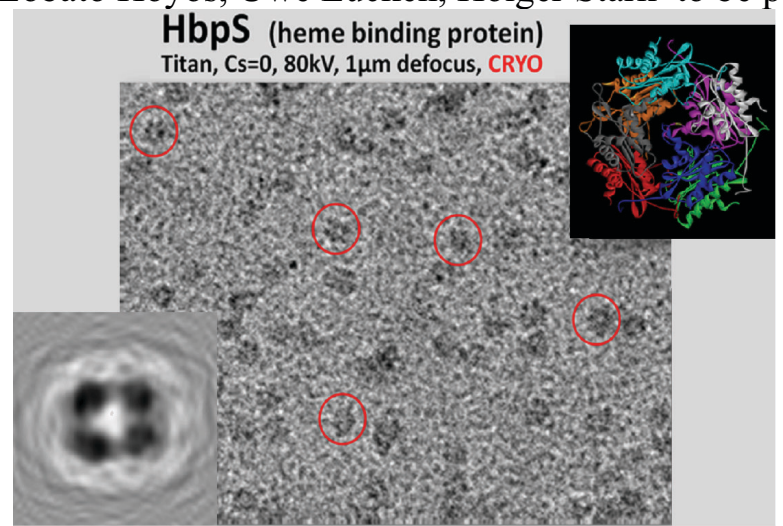

Fig.1: Cs corrected cryo-EM image of $\mathrm{HbpS}$ proteins with remarkably high contrast for small particles.
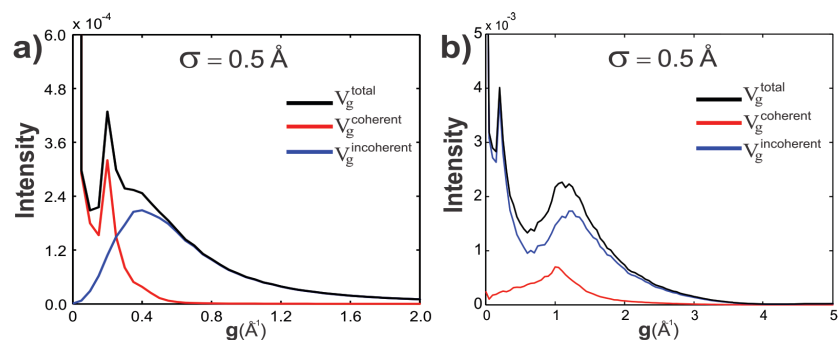

Fig. 2. Rotationally averaged power spectra of a frozen atom image calculation of an $\mathbf{H b p S}$ protein at $300 \mathrm{KeV}$. a) Diffraction and b) Diffractogram for an electron microscope parameters $(\Delta=3.2 \mathrm{~nm}$, $\left.\alpha=0.2 \mathrm{mrad}, \mathrm{C}_{\mathrm{s}}=0.04 \mathrm{~mm}, \Delta \mathrm{f}=10.9 \mathrm{~nm}\right)$ with annular dark-field aperture $\left(\mathrm{g}_{\min }=0.5 \AA^{-1}, \mathrm{~g}_{\max }=2.0^{-1}\right)$. Total(black curve), coherent(red curve)and incoherent(blue curve) RAPS for RMS $=0.5 \AA$.
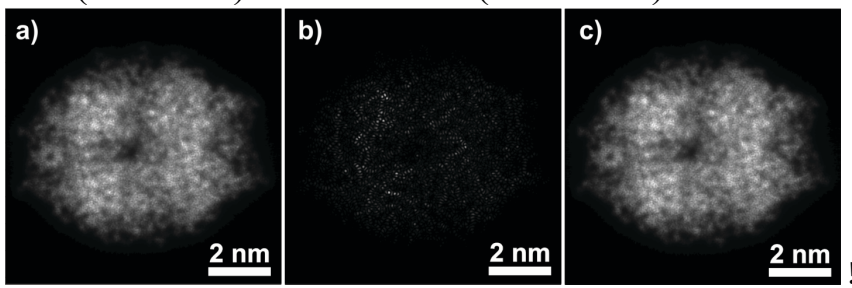

Fig. 3. Frozen atom HREM image calculation of an $\mathbf{H b p S}$ protein with annular dark-field aperture $\left(\mathrm{g}_{\min }=0.5 \AA^{-1}, \quad \mathrm{~g}_{\max }=2.0 \AA^{-1}\right)$. Electron microscope parameters: acceleration voltage $\left(\mathrm{E} 0=300 \mathrm{KeV}, \Delta=3.2 \mathrm{~nm}, \alpha=0.2 \mathrm{mrad}, \mathrm{C}_{\mathrm{s}}=0.04 \mathrm{~mm}, \Delta \mathrm{f}=10.9 \mathrm{~nm}\right)$. a) Total intensity, b) Coherent intensity contribution and c) Incoherent intensity contribution. 